\title{
Supporting Information for: Small-angle neutron scattering (SANS) characterization of clay- and carbonate-rich shale at elevated pressures
}

Chelsea W. Neil ${ }^{1,}$, Rex P. Hjelm², Marilyn E. Hawley ${ }^{3}$, Erik Watkins ${ }^{4}$, Cody Cockreham ${ }^{1,5}$, Di $\mathrm{Wu}^{5,6,7,8}$, Yimin $\mathrm{Mao}^{9}$, Timothy B. Fischer ${ }^{10}$, M. Rebecca Stokes ${ }^{10}$, and Hongwu Xu ${ }^{1}$

${ }^{1}$ Earth and Environmental Sciences Division, Los Alamos National Laboratory, Los Alamos, NM 87545, USA

${ }^{2}$ National Security Education Center, Los Alamos National Laboratory and the New Mexico Consortium, Los Alamos, New Mexico 87545, USA

${ }^{3}$ Retired: Materials Science and Technology Division, Los Alamos National Laboratory, Los Alamos, New Mexico 87545, USA

${ }^{4}$ Materials Physics and Applications Division, Los Alamos National Laboratory, Los Alamos, New Mexico 87545, USA

${ }^{5}$ Voiland School of Chemical Engineering and Bioengineering, Washington State University, Pullman, WA 99164, USA

${ }^{6}$ Alexandra Navrotsky Institute for Experimental Thermodynamics, Washington State University, Pullman, WA 99164, USA

${ }^{7}$ Materials Science and Engineering, Washington State University, Pullman, WA 99164, USA

${ }^{8}$ Department of Chemistry, Washington State University, Pullman, WA 99164, USA

${ }^{9}$ NIST Center for Neutron Research, National Institute of Standards and Technology, Gaithersburg, Maryland 20899, USA

${ }^{10}$ Chevron Energy Technology Company, 3901 Briarpark Drive, Houston, Texas 77042, USA

Address: P.O. Box 1663, Los Alamos, NM 87545

E-mail: cwneil@lanl.gov

Phone: (908)-489-4463

Submitted: October 2019, Revised: March 2020, May 2020

Energy \& Fuels

*To Whom Correspondence Should be addressed

7 pages, including 8 figures. 

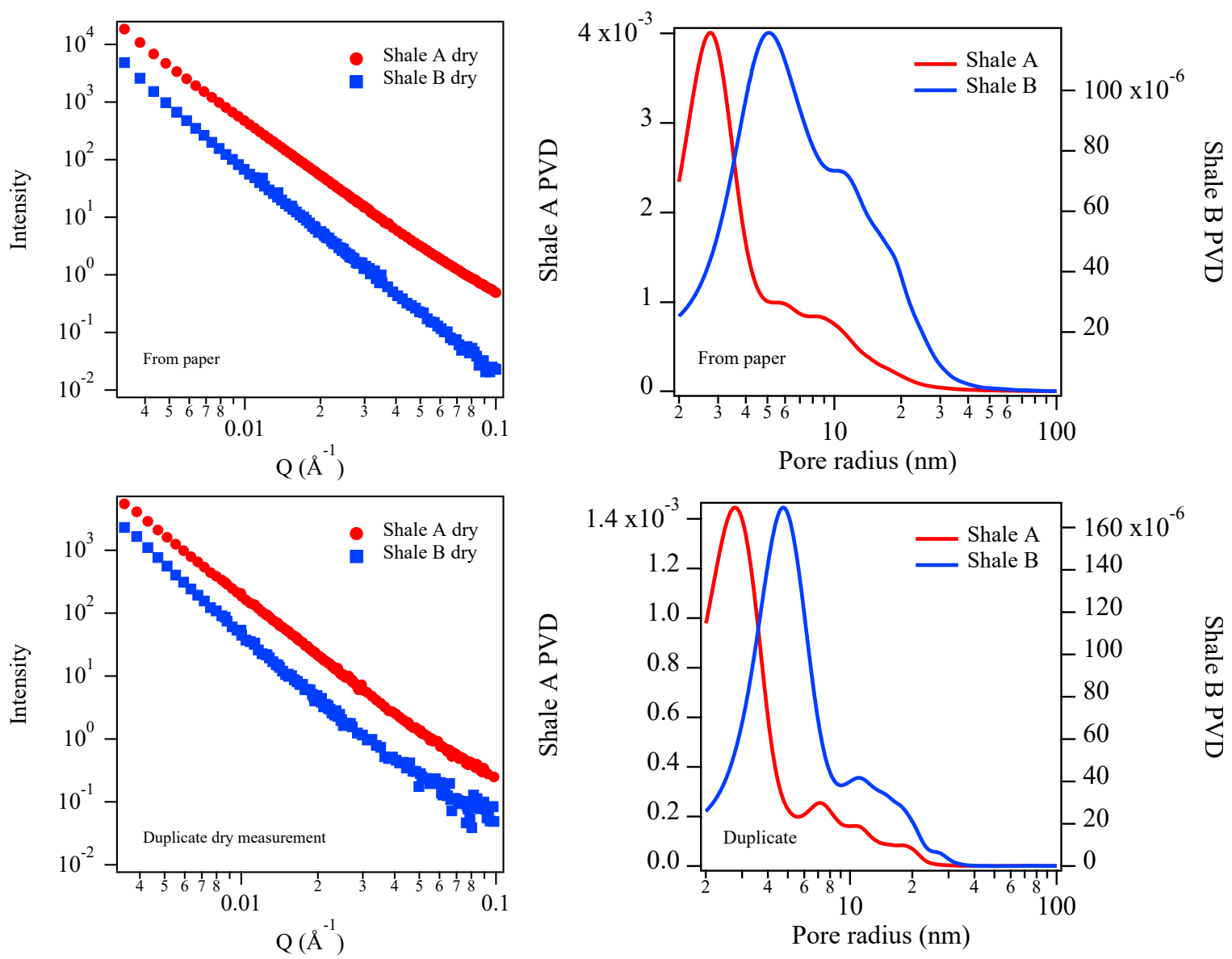

Figure S1. Duplicate dry measurements show consistent intensity differences and dominant pore sizes.

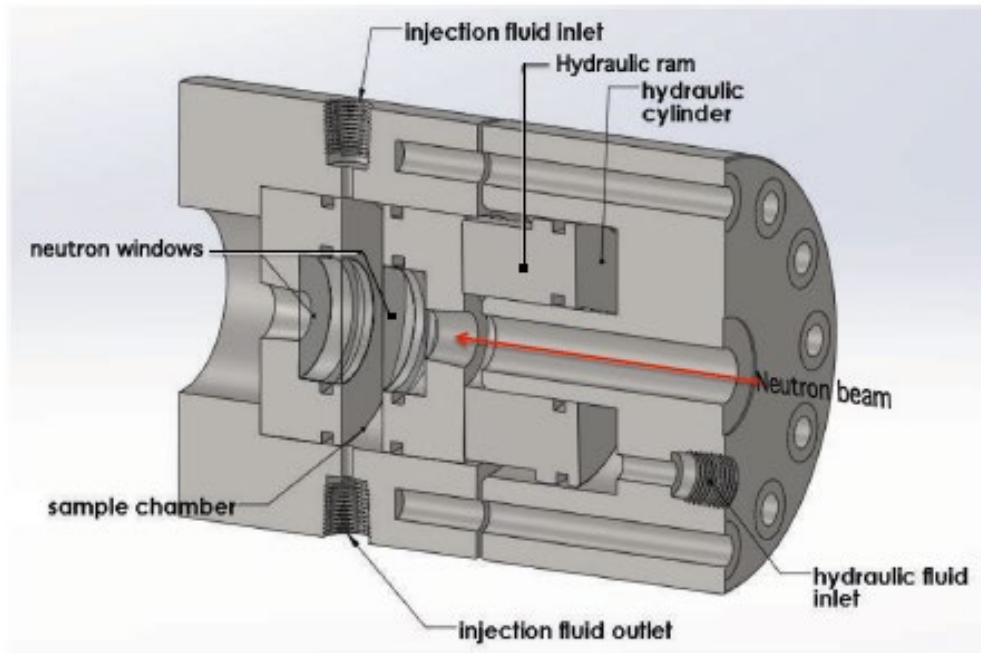

Figure S2. SANS compression cell schematic (from Hjelm et al., 2018) 

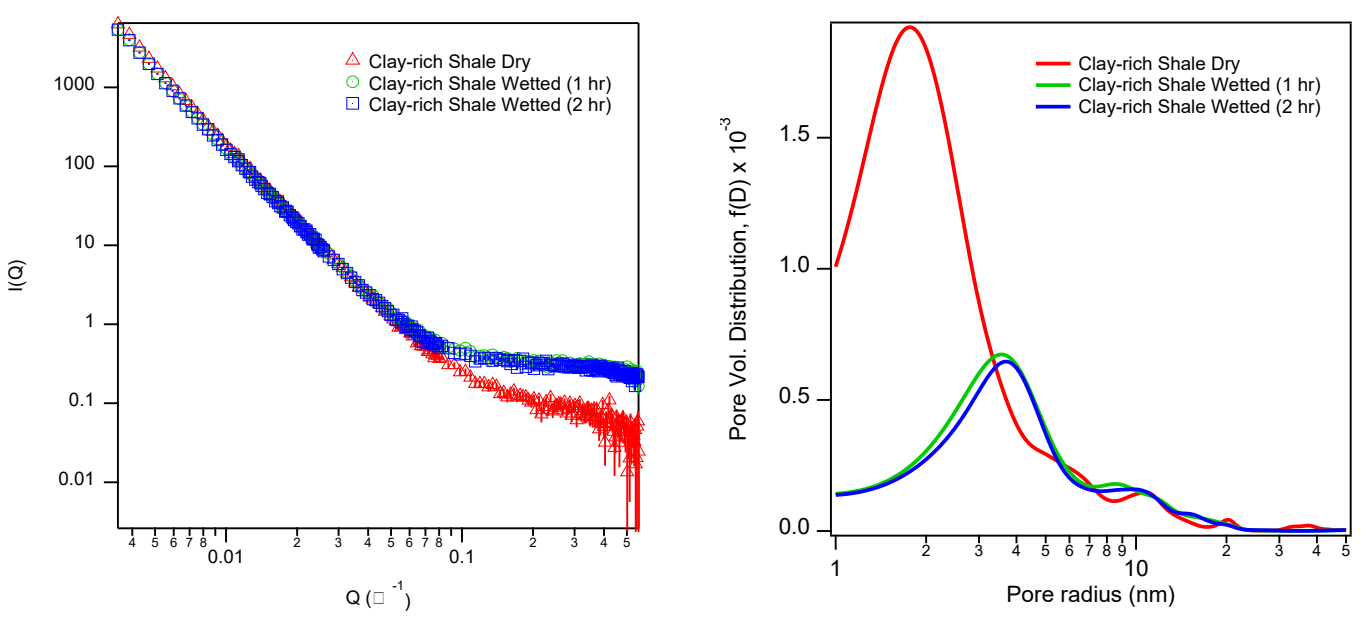

Figure S3. Saturation testing shows the $300 \mu \mathrm{m}$ shale sample saturates within 5 minutes

A. Shale A (clay-rich)

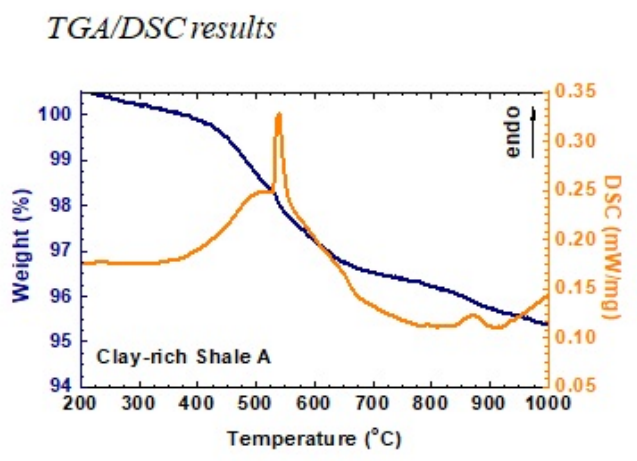

XCT results

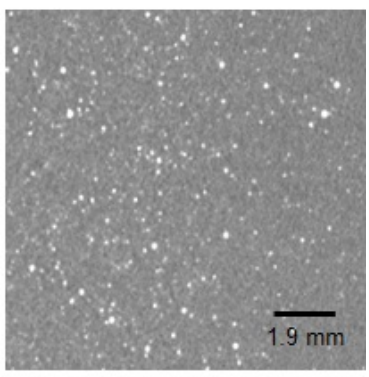

B. Shale B (carbonate-rich)

\section{TGA/DSCresults}

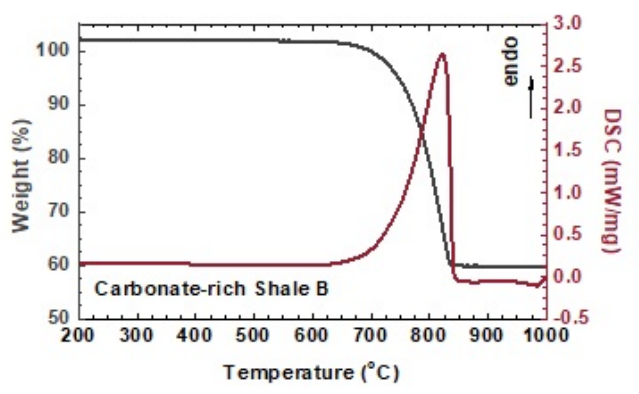

XCT results

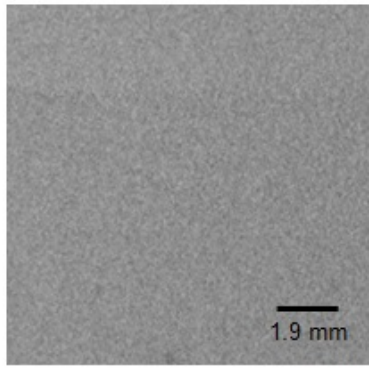

Figure S4. TGA/DCS and XCT images of clay-rich (A) and carbonate-rich (B) shale samples 

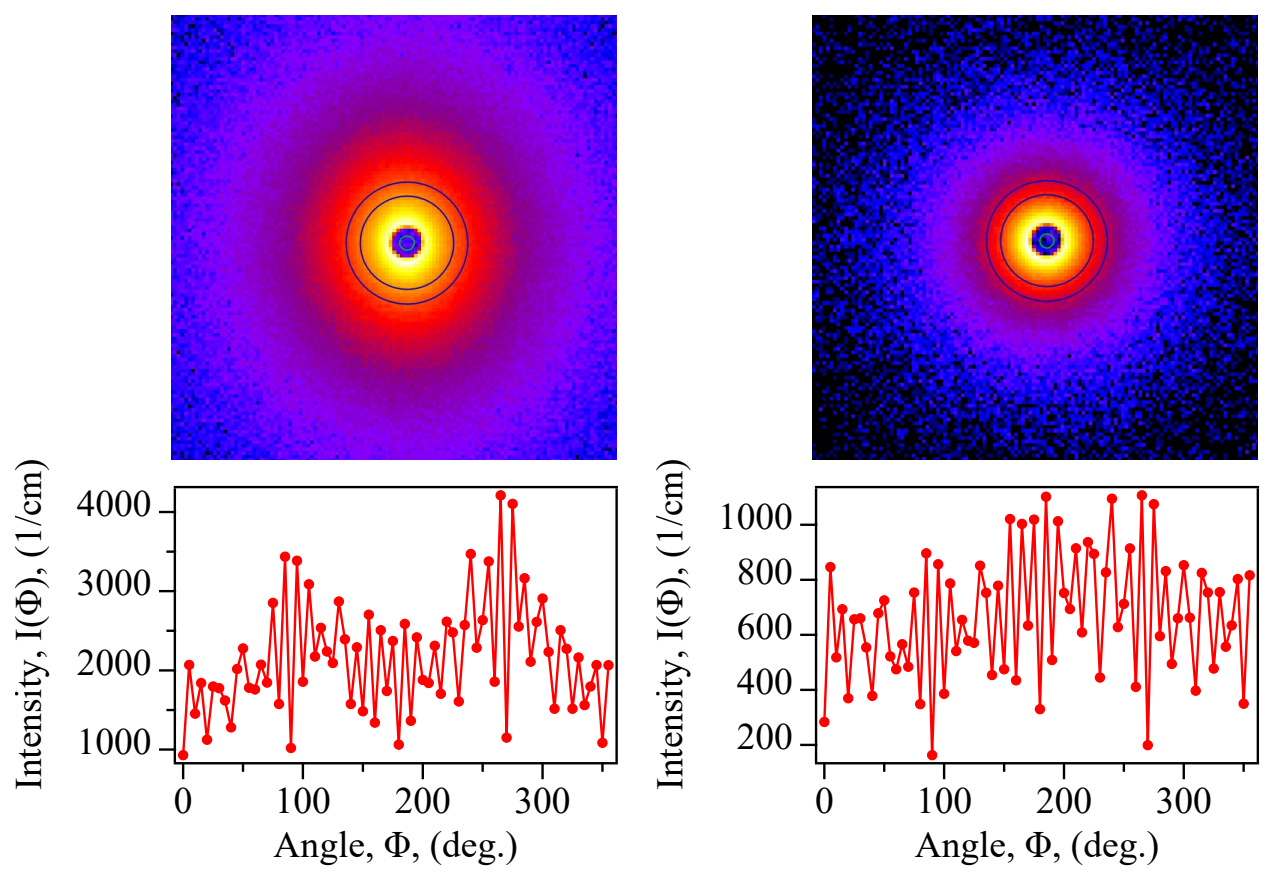

Figure S5. 2D SANS scattering and intensity as a function of azimuthal angle for Shale A and B samples 

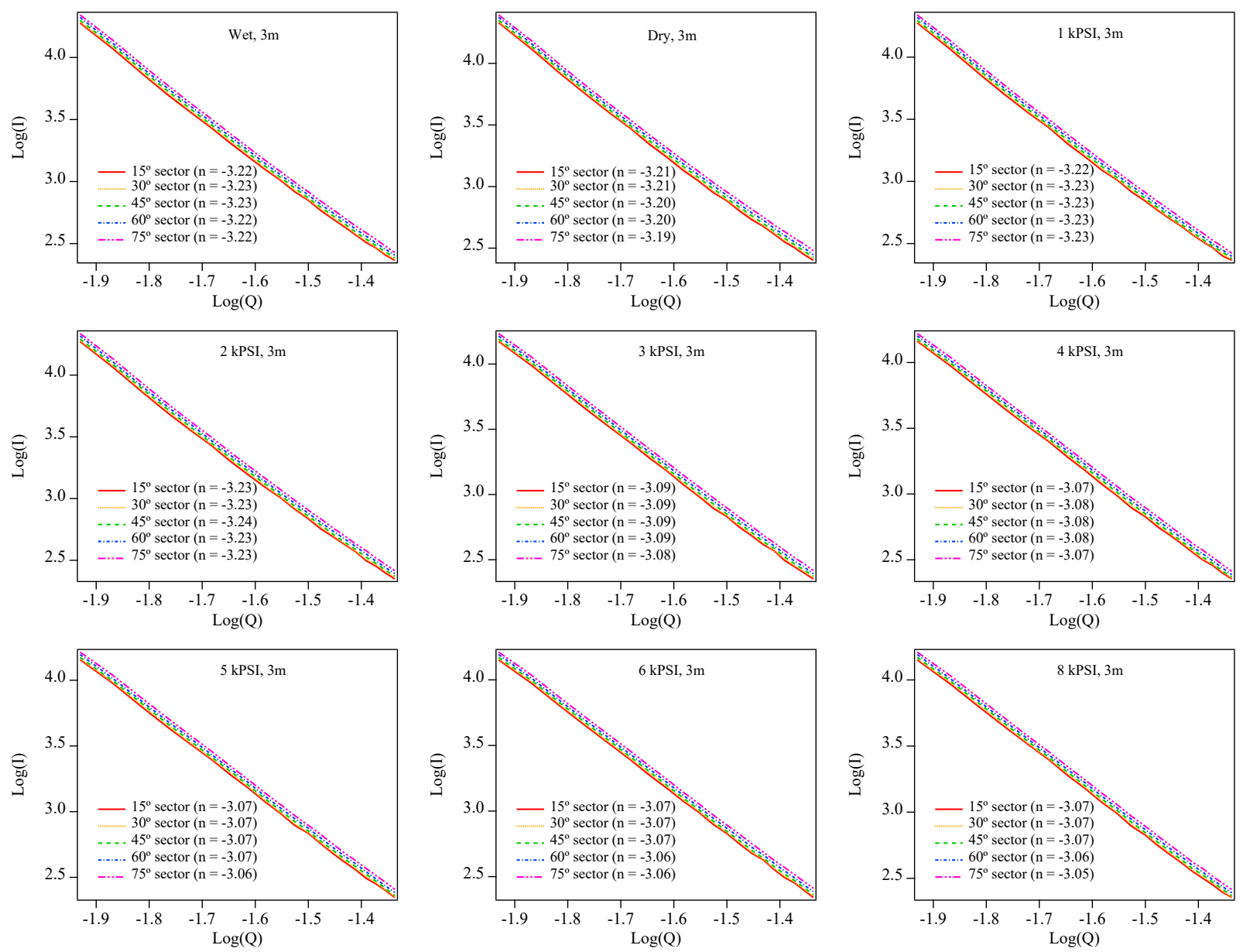

Figure S6. $\log (\mathrm{I})$ vs. $\log (\mathrm{Q})$ taken at $15^{\circ}$ section increments for the Shale A scattering profiles at a 3 m sample-to-detector distance. Power law fitting for these low $Q$ regions did not change significantly as the section changed. The intensity offset is a result of the anisotropic nature of the samples. 

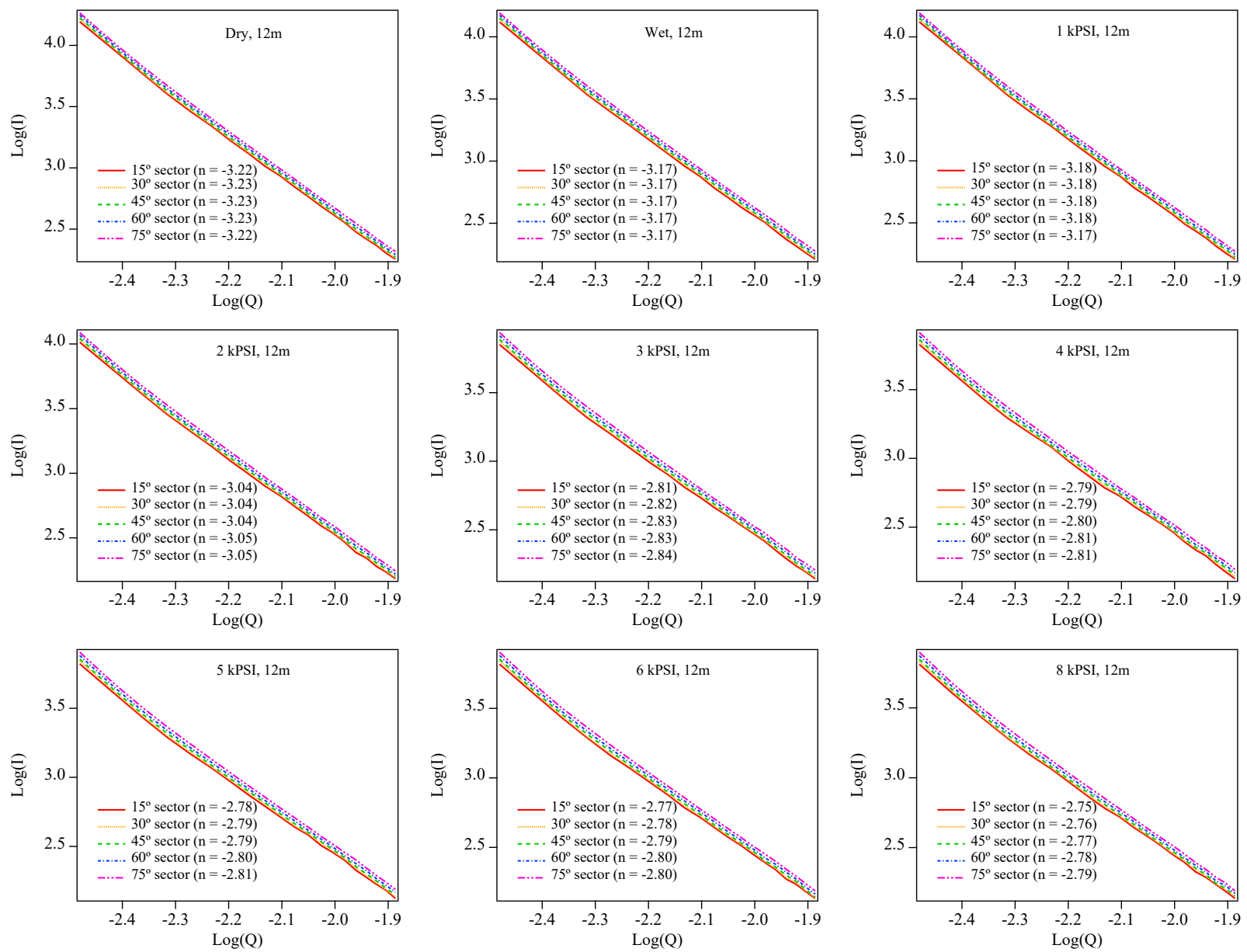

Figure S7. $\log (\mathrm{I})$ vs. $\log (\mathrm{Q})$ taken at $15^{\circ}$ section increments for the Shale A scattering profiles at a 12 m sample-to-detector distance. Power law fitting for these low $Q$ regions did not change significantly as the section changed. The intensity offset is a result of the anisotropic nature of the samples. 

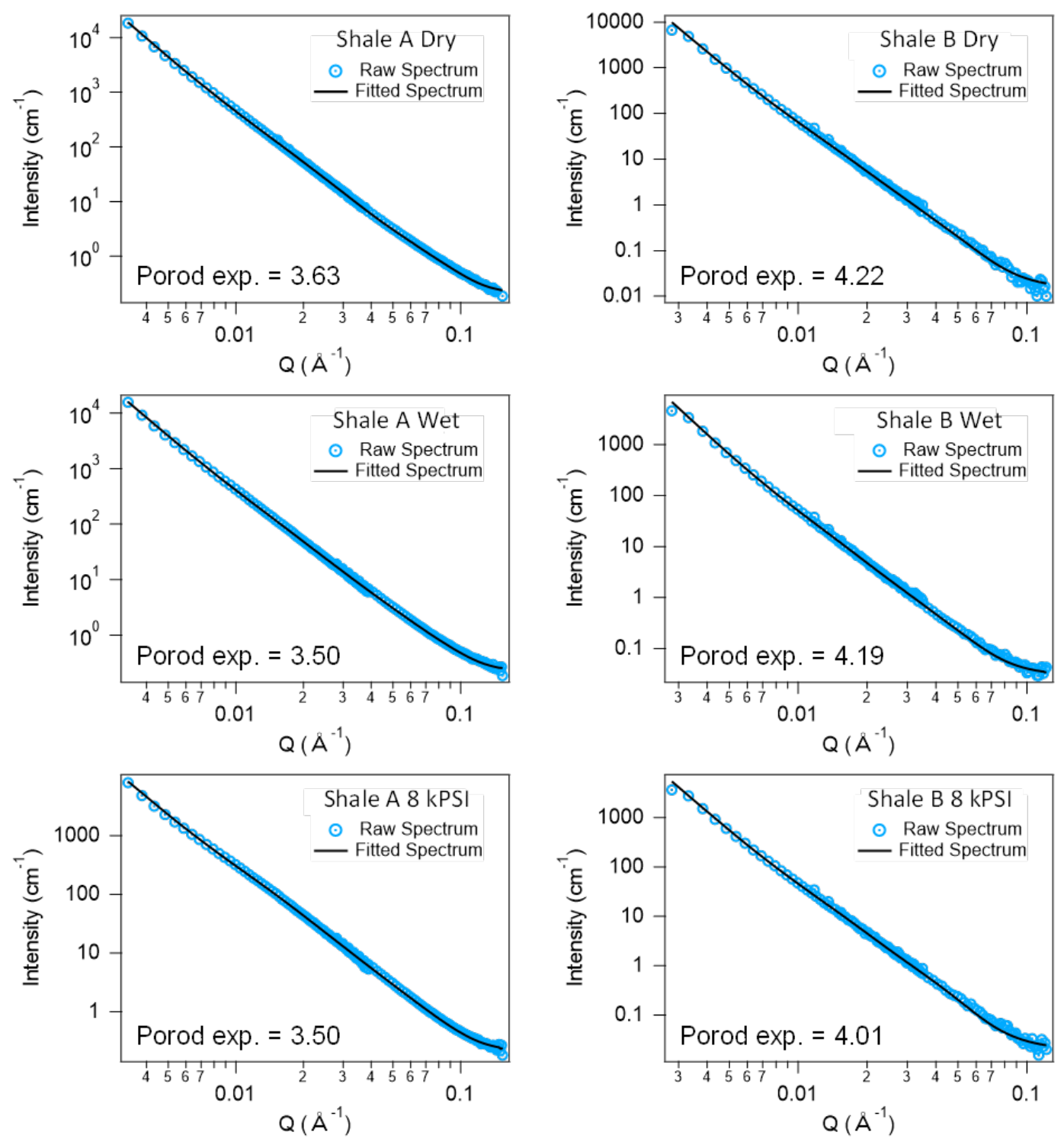

Figure S8. Scattering profiles and profiles fitted using the calculated pore size distributions.

\section{References}

Hjelm, R.P., Taylor, M.A., Frash, L.P., Hawley, M.E., Ding, M., Xu, H., Barker, J., Olds, D., Heath, J. and Dewers, T., 2018. Flow-through compression cell for small-angle and ultra-smallangle neutron scattering measurements. Review of Scientific Instruments, 89(5), p.055115. 